Z. klin. Chem. u. klin. Biochem.

7. Jg., S. 275-277, Mai 1969

\title{
Antigeneigenschaften des C-reaktiven Proteins
}

\author{
Von Hilde Götz, M. Pérez-Miranda und F. Scheiffarth \\ Abteilung für klinische Immunologie des Universitätskrankenbauses Erlangen-Nürnberg \\ (Vorstand: Prof. Dr. F. Scheiffarth)
}

(Eingegangen am 27. Dezember 1968)

\section{Herrn Prof. Dr. H. E. Bock zum 65. Geburtstag genvidmet}

Immunchemische Untersuchungen an 70 Seren von Gesunden und 25 Patientenseren zum Nachweis des C-reaktiven Proteins haben zu folgenden Ergebnissen geführt: Das C-reaktive Protein ist in allen normalen Seren nachweisbar. Es zeigt mit keinem der bekannten Immunglobuline des menschlichen Serums immunologische Identitäts- oder Verwandtschaftsreaktionen. Es findet sich auf Grund seiner immunoelektrophoretischen Position im Bereich der $\boldsymbol{\gamma}$-Immunglobuline. Es scheint als unspezifischer Abwehrkörper im Initialstadium eines Infekt- oder Inflammationsgeschehens im Sinne einer Sofortaggression aktiviert zu werden und hat somit teil an den Abwehrmaßnahmen des Organismus.

\section{Antigenic properties of $C$-reactive protein}

Immunochemical analyses of 70 sera of healthy persons and of 25 patient's sera for C-reactive protein have shown the following results: C-reactive protein is demonstrable in all normal sera. It dosn't show identical or related immunological reactions with any of the well known immunoglobulins of human serum. According to its immunoelectrophoretic position it belongs to the class of gamma globulins. It seems to be activated as an unspecific antibodylike factor in the initial phase of infectious or inflammatory processes in a sense of an acute aggression; therefore it participates in the defensive proceedings of an organism.

In bisherigen eigenen Mitteilungen über den Nachweis des C-reaktiven Proteins (CRP) in Normalseren $(1,2)$ wurde festgestellt, daß das CRP bei insgesamt 50 Seren von gesunden Blutspendern in $100 \%$ der Fälle nachgewiesen werden konnte, daß dieses mit dem stark vermehrten $\mathrm{C}$-reaktiven Protein CRP-positiver $\mathrm{Pa}$ tientenseren immunologische Antigenidentität zeigt, und daß das CRP-Präzipitat der Normalseren immunoelektrophoretisch mit dem Präzipitationsphänomen der pathologischen Seren überreinstimmt.

In den vorliegenden Untersuchungen sollten Beziehungen des CRP zu den normalen $\gamma$-Immunglobulinklassen $\operatorname{IgG}, \operatorname{Ig} A, \operatorname{IgM}$ sowie $\operatorname{IgD}$ aufgeklärt werden mit der Frage nach Antigengemeinschaften mit diesen Immunglobulinen bzw. nach einer antigenen Unabhängigkeit von den bekannten $\gamma$-Globulinen.

\section{Versuchsanordnung und Methodik \\ Versuchsanordnung}

Es wurden insgesamt 70 Normalseren ${ }^{1}$ ) sowie 25 CRP-positive ${ }^{2}$ ) Patientenseren untersucht. Jedes Serum wurde mit der zweidimensionalen Immunodiffusion nach OuCHTERLONY mit einem handelsüblichen Anti-CRP-Serum ${ }^{3}$ ) sowie mit monoclonalen Antiseren gegen die Immunglobuline IgA, IgG, IgM und IgD getestet $\left.^{3}\right)$. Außerdem wurde jede Serumprobe immunoelektrophoretisch analysiert.

1) Die Seren verdanken wir der Zentralen Blutbank der Universitätskliniken Erlangen-Nürnberg (Leiter: Dr. $K$. Trr. SCHRICKER).

2) Es handelt sich um mit der üblichen Latex-CRP-Nachweistechnik ermittelte CRP-positive Patientenseren. CRP-LatexReagenzien d. Fa. Behringwerke, Marburg/Lahn.

3) Anti-CRP-Serum sowic die Antiseren gegen die genannten Ig-Systeme wurden sämtlich von den Behringwerken, Marburg/ Lahn bezogen. Antiserum gegen IgE stand leider nicht zur Verfügung.
Tab. 1

Erforderliche Konzentrationen bzw. Verdünnungen der Antiseren sowie der $z u$ testenden Normal- und Patientenseren. $1: 1=$ unverdünntes Antiserum, entsprechend der handelsüblichen Konzentration

\begin{tabular}{lccc}
\hline & Antiseren & $\begin{array}{c}\text { Optimale Verdünnung } \\
\text { Normalseren }\end{array}$ & $\begin{array}{c}\text { CRP-positive } \\
\text { Seren }\end{array}$ \\
\hline Anti-IgA & $1: 1$ & $1: 8$ & $1: 8$ \\
Anti-IgG & $1: 1$ & $1: 8$ & $1: 8$ \\
Anti-IgM & $1: 2$ & $1: 4$ & $1: 4$ \\
Anti-IgD & $1: 8-1: 16$ & $1: 4$ & $1: 4$ \\
Anti-CRP & $1: 8-1: 16$ & $1: 1-1: 2$ & $1: 4-1: 8$ \\
\hline
\end{tabular}

Methodik

Zweidimensionale Immunodiffusion im Agargel

Es wurde die bekannte Technik nach OuchrerLoNy (3) gewählt. Dabei wurde der gepufferte Agar in einer Konzentration von $0,7 \%$ verwendet. Die Serumproben bzw. spezifischen Antiseren wurden entsprechend den Angaben der Tabelle 1 angesetzt. Die Inkubationszeit betrug bei $37^{\circ} 24$ bis $48 \mathrm{Stdn}$.

\section{Immunoelektrophorese}

Es wurde die Methode nach Grabar und Williams (4) mit 0,7proz. gepuffertem Agar und Agarplatten mit den Abmessungen $9 \times 12 \mathrm{~cm}$ (für Multianalysen) angewandt. Die Serumproben der Gesunden gelangten in einer 4- bis $8 \mathrm{mal}$ stärkeren Konzentration als der Normalkonzentration zur Analyse, die CRP-positiven Seren in Normalkonzentration. Die Verdünnung des Anti-CRPSerums betrug zwischen $1: 8$ und $1: 16$, in Einzelfällen $1: 32$. Zwölf der CRP-positiven Seren wurden darüber hinaus mit einem polyvalenten Antihumanserum ${ }^{4}$ ) in einer Verdünnung 2:3 immunoelektrophoretisch analysiert.

\section{Ergebnisse}

Ergebnisse mit der qweidimensionalen Immunodiffusion

Die vergleichenden Untersuchungen des CRP-AntiCRP-Systems mit den bekannten Immunglobulinen des

4) Antihumanserum von der Ziege (polyvalentes Antibumanserum) der. Fa. Hyland Laboratories Los Angeles, Vertrieb Travenol International, München. 

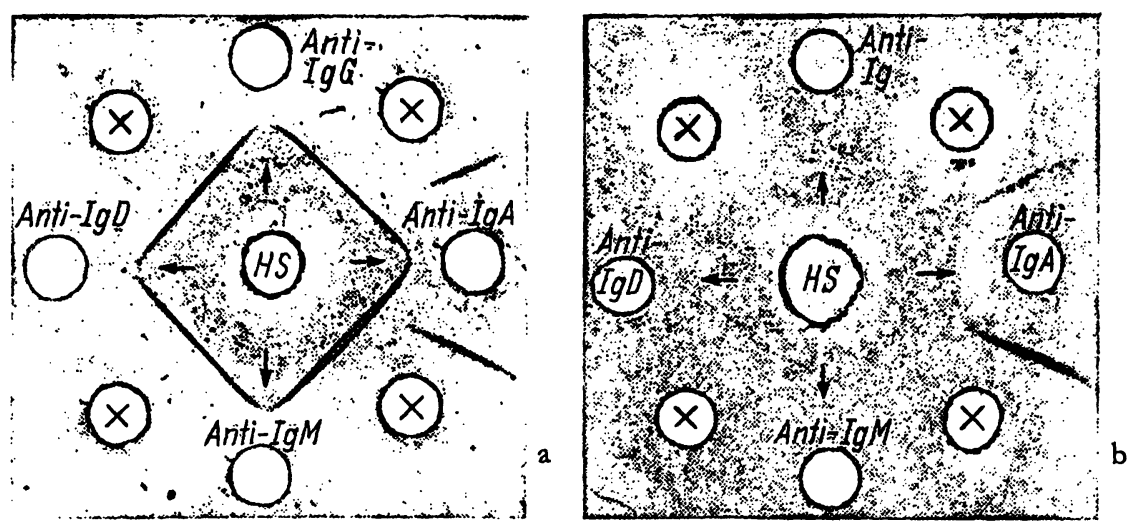

Abb. 1

Ergebnisse der zweidimensionalen Immunodiffusion im Agargel zum Ausschluß von immünologischen Verwandtschafts- oder IdentiKlassen der normalen Immunglobuline des Klassen der normalen Immunglobuline

HS = Humanserum (Normalserum)

$\mathbf{x}=$ Anti-CRP-Serum

$a$ und $b$ geben zwei verschiedene Analysensätze mit unterschiedlichen Verdünnungsgraden von Serum und Antiseren wieder menschlichen Serums ergaben für die Ig-Klassen IgA, IgG, IgM sowie IgD immunologisch total gekreuzte Präzipitationsphänomene, d. h. Fremdreaktion (Abb. 1a, b).

\section{Immunoelektrophoretische Ergebnisse}

Die immunoelektrophoretischen Befunde zeigten bei 67 von 70 der untersuchten Blutspenderseren und bei 20 von den 25 pathologischen, CRP-positiven Seren übereinstimmend eine relativ langgestreckte, schwach gebogene:Präzipitationslinie, die geringfügig unterhalb $\operatorname{der} \gamma$ G-Linie verläuft (Abb. 2), und die auch in den mit polyvalentem Antihumanserum geprüften CRP-positiven Seren zur Darstellung gelangte (Abb. 2). In den verbliebenen 7 Fällen trat eine deutliche Doppelung

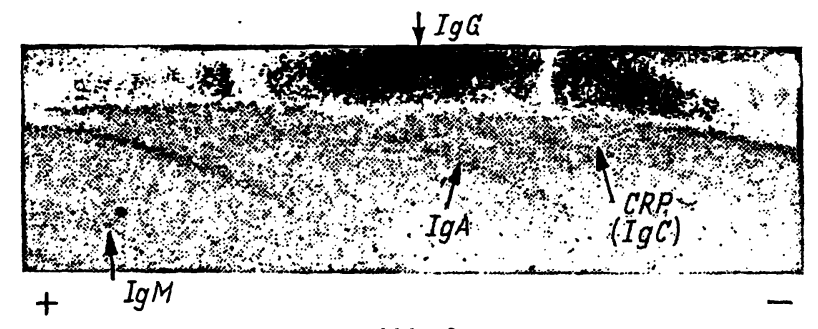

Abb. 2

Immunoelektrophorese eines CRP-positiven Patientenserums unter Verwendung eines polyvalenten Antihumanserums. Man erkennt relativ deutlich an dem vergrößerten Ausschnitt des $\gamma$-Globulin-
Systems die langgestreckte CRP-Linie dicht unterhalb des IgGPräzipitats

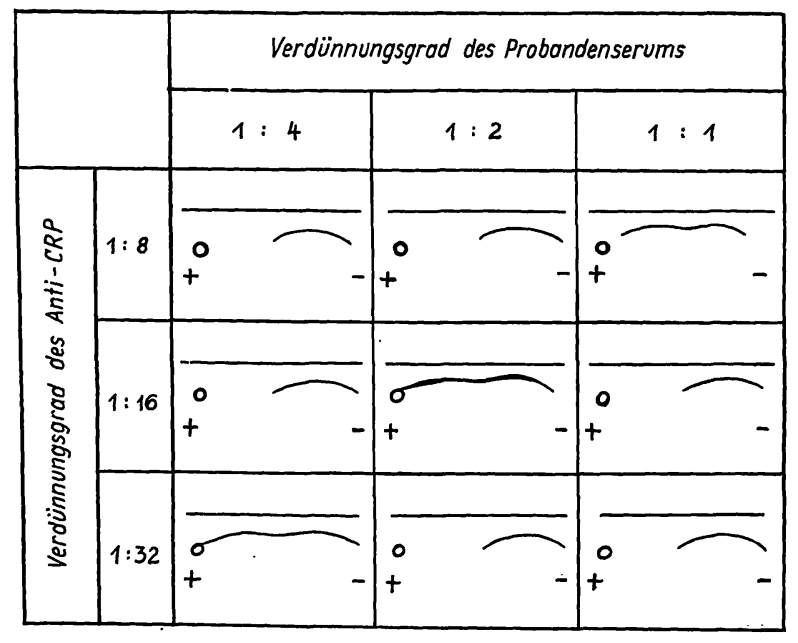

Abb. 3

Halbschematische Darstellung verschiedener Präzipitationsphänomene des CRP aus einem Analysensatz von Immunoelektrophoresen mit schiedenen Verdünnungen unter Verwendung verschiedener Verdünnungen auch des Anti-CRP-Serums
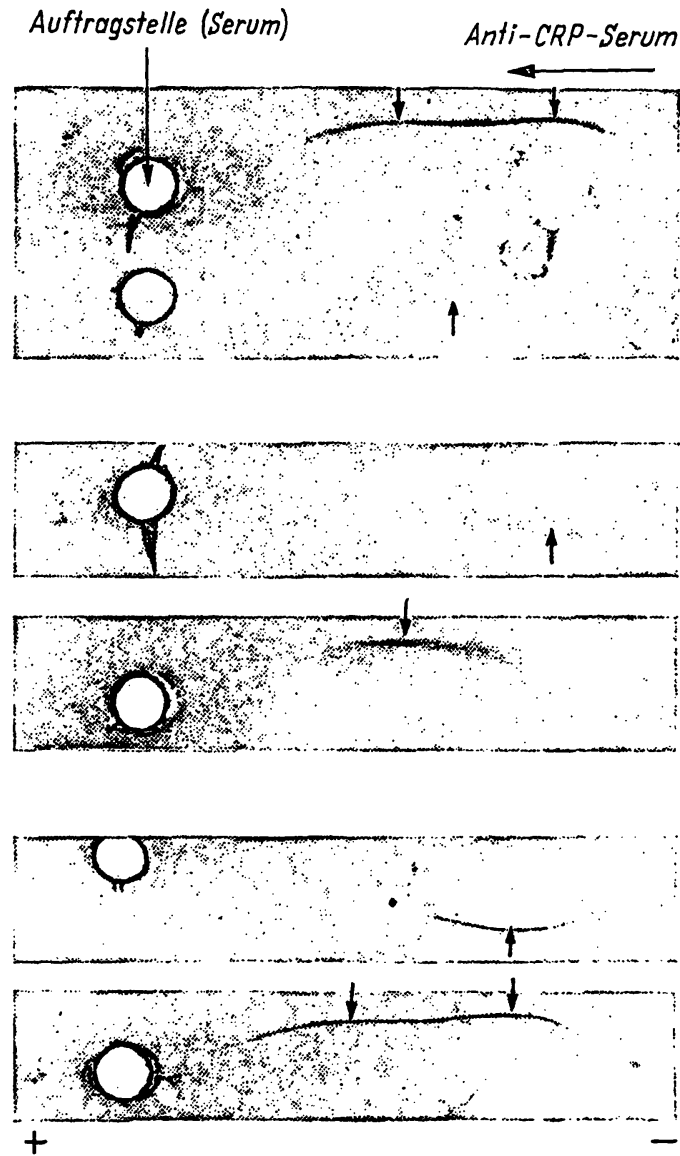

Abb. 4

Immunoelektrophoretisch verschieden darstellbare CRP-Präzipitate ein und desselben CRP-positiven Patientenserums. Serum und AntiSerum wurden in verschiedenen Verdünnungen angesetzt. Vgl. auch Abbildung 3

der CRP-Linie in Erscheinung (Abb. 3 u. 4). Studien mit CRP-positiven Seren in unterschiedlicher Konzentration bzw. unterschiedlicher Verdünnung ergaben folgendes (Abb. 3 u. 4): Es konnte festgestellt werden, $\mathrm{da} \beta$ mit ein und demselben Serum bei verschiedener Serum- und Antiserumkonzentration unterschiedliche Präzipitationsphänomene für das CRP-Anti-CRP-System zur Darstellung gelangten.

\section{Diskussion}

Die Ergebnisse der vorliegenden Immunodiffusionsanalysen des spezifischen CRP-Systems mit den Systemen der bekannten Immunglobuline IgA, IgG, IgM sowie IgD haben gezeigt, daß das ${ }_{i} \mathrm{CRP}$ der Normalseren 
ebenso wie das der CRP-positiven Seren mit keinem der getesteten klassenspezifischen Immunglobuline eine immunologische Identität, nicht einmal Teilidentität aufweisen. Diese Tatsache und außerdem der regelmäßige Nachweis des CRP in Normalseren berechtigt zur Annahme, daß es sich bei dem CRP nicht um ein pathologisches Globulin sondern um ein Normalprotein etwa vom Typ der Immunglobuline handelt. Wir haben bereits in früheren Mitteilungen $(1,2)$ den Vorschlag unterbreitet, dieses als IgC zu benennen.

An die Möglichkeit, daß es sich bei dem CRP auch um ein Normalglobulin handeln könnte, etwa um ein Transportglobulin für Fremdantigene, wurde bereits von Parker, Stackiw und Wilt (6) gedacht. Der Nachweis hierfür war jedoch bislang nicht erbracht worden.

Die immunoelektrophoretischen Befunde stimmen gut überein mit den Ergebnissen der einschlägigen Literatur, die allerdings ausschließlich an pathologischen, d. h. CRP-positiven Seren gewonnen wurden $(7,8,9)$. Sie unterscheiden sich hinsichtlich der Zuordnung innerhalb der Serumkonstituenten des menschlichen Serums von früheren Angaben $(10,11,12)$. Wir haben früher selbst darauf hingewiesen, daß unter Verwendung eines polyvalenten Anti-Normalserums das CRP in pathologischen Seren ohne weiteres zur Darstellung gebracht werden kann (13, 14), eine Beobachtungstatsache, die sich mit den vorliegenden Ergebnissen bestätigen ließ, und die außerdem beweist, daß in den zur Sensibilisierung verwendeten Seren zur Gewinnung der polyvalenten Antihumanseren das CRP enthalten gewesen sein mußte. Wir haben damals allerdings nicht den naheliegenden Schluß gezogen, daß es sich deshalb bei dem CRP um ein Normalprotein handeln müßte. Inwieweit die vorliegenden Ergebnisse mit Bezug auf das in der Literatur beobachtete $(15,16,17)$ und auch von uns festgestellte Doppelungsphänomen der CRPLinie eine Klärung darstellen, kann vorerst noch nicht entschieden werden. Nach den eigenen Analysen mit Serumproben in verschiedenen Konzentrationen bzw. Verdünnungen handelt es sich bei den unterschiedlich in Erscheinung tretenden Präzipitationslinien bzw. CRP-Komponenten um konzentrationsabhängige Phänomene. Die Entscheidung darüber, ob gleichzeitig damit qualitative Unterschiede innerhalb des CRPSystems verbunden sind, wie dies RILEY, Coleman und HoкAma (18) zu beweisen geglaubt haben, oder ob es sich bei dem CRP um zwei nach ihrer elektrophoretischen Beweglichkeit verschiedene, immunologisch jedoch identische Komponenten handelt, wie dies Anzai, Sato, Fukuda und Carpenter (19) annehmen, wird wohl weiteren Analysen an gereinigten CRPPräparaten vorbehalten bleiben. Analoge Präzipitationsphänomene finden sich im übrigen auch bei anderen Normalproteinen, man denke etwa an die $\tau$-Fraktion des Liquor cerebrospinalis oder an die Transferrine des menschlichen Serums.

Insgesamt haben die vorliegenden Untersuchungsergebnisse an normalen wie CRP-positiven, pathologischen Seren es wahrscheinlich gemacht, daß das CRP als ein physiologischer Serumeiweißkörper aufzufassen ist.

\section{Literatur}

1. Pérez-Miranda, M. und H. Götz, Lancet (London) 1968/II, 1305. - 2. Scheiffarth, F., M. Pérez-Miranda und H. Götz, Clin. Chim. Acta Amsterdam (zum Druck angeboten). - 3. OucrTERlonY, Ö., Progr. Allergy 5, 1. S. Karger, Basel (1958). - 4. Grabar, P. und C. A. Williams, Biochim. biophysica Acta Amsterdam 10, 193 (1953). - 5. Grabar, P. und P. Burtin, Immunoelektrophoretische Analyse, Elsevier Publ. Comp. Amsterdam (1964). - 6. Parker, W. L., W. Stackrw und J. C. WiLt, Canad. Med. Ass. J. 87, 791 (1962). - 7. FerRr, R. G. und W. Cossermelil, Arch. Internat. Rheumatol. 1, 493. (1958). - 8. Schultze, H. E., G. Schwick, J. Sonnet, J. Heremans und J. L. MichauX, Klin. Wschr. 38, 62 (1960). - 9. ZACH, J. und K. ZimmermanN, Klin. Wschr. 37, 160 (1959). - 10. Burtin, P., Sem. hôp. (Paris) 33, 2177 (1957). - 11. Bustamante, V., J. Arino und L. Manued Y Puniès, Presse méd. Paris 65, 313
(1957). und Rev. clin. espan. 67, 9 (1957). - 12. Gautrer, A. und J. J. Scheidegger, Schweiz. med. Wschr. 87, 950 (1957). 13. Scheiffarth, F. und H. Götz, in Henning, N. Praktische Etgebnissse neuer klinischer Forschung, S. 15, Schattauer, Stuttgart (1962). - 14. Scheiffarth, F., H. GötZ und E. BaIGger, Med. Welt 51, 2611 (1963). - 15. Hokanra, Y. und R. F. Riley, Biochim. biophysica Acta Amsterdam 74, 305 (1963). - 16. Libretri, A., M. A. Kaplan und M. Goldin, Proc. Soc. exper. Med. Biol. 90, 481 (1955). - 17. Nakamura, R. M., R. Magsaysay, J. Ford und G. M. Kunitaki, Amer. J. Clin. Path. 44, 290 (1965). 18. Rilex, R. F., M. K. Coleman und Y. Hokama, Clin. Chim. Acta Amsterdam 11, 530 (1965). - 19. AnzaI, T., K. Sato, M. Fukuda und C. M. Carpenter, Proc. Soc. exper. Med. Biol. 120, 94 (1965).
Priv. Doz. Dr. Hilde Götz 8520 Erlangen Krankenhausstraße 12 